\title{
Preventative and Personalized Approach to the Treatment of Malignant Melanoma: A Case Report
}

\author{
JD Matthew Hughes $B S c(H)^{1}$, Libni J. Eapen, $M D, F R C P(C)$, $F A C R^{2}$, Jennifer Beecker, $M D, F R C P(C)^{3}$ \\ ${ }^{1}$ Faculty of Medicine, University of Ottawa \\ ${ }^{2}$ The Ottawa Hospital Cancer Program \\ ${ }^{3}$ Division of Dermatology, Department of Medicine, The Ottawa Hospital
}

\section{A BSTRACT}

This case report is focused on malignant melanoma, a common potentially lethal skin cancer, and its unique management. In our patient's case, the primary cutaneous melanoma occurred 28 years prior to presenting with axillary lymphadenopathy, which was later determined to be a metastatic focus of melanoma; subsequent nodules were found in his spleen. After completion of his treatment and routine screening, additional nodules were discovered in his chest. Primary and secondary prevention were important in the management of this patient, as well as personalization of his treatment.

\section{RÉSUMÉ}

Cette étude de cas porte sur le mélanome malin, un cancer de la peau potentiellement mortel avec une gestion unique. Dans le cas de notre patient, le mélanome s'est présenté 28 ans avant la présentation d'une lymphadénopathie axillaire, ce qui a été démontré par la suite comme une zone étant métastatique du mélanome. Des nodules ont été découverts dans sa rate. Suite à la fin des traitements et des suivis de routine, des nodules additionnels ont été découverts dans sa poitrine. Des préventions primaires et secondaires sont importantes pour la gestion de ce patient ainsi qu'un traitement personnalisé.

\section{INTRODUCTION}

Malignant melanoma is a common, potentially lethal skin cancer [1] with nearly 1,150 deaths and 6,800 new cases in Canada in 2015 [2]. Breslow depth is used as a prognostic measure where stage I cases ("thin melanomas") can expect long-term survival or cure, but thicker tumours $(>2.0 \mathrm{~mm}$ ) have poorer prognosis [3]. In fact, greater depth, presence of ulceration or mitoses, positive lymph nodes, including clinically unapparent lymph nodes, and visceral metastases are all associated with a worse prognosis [3]. The five-year survival over all stages of melanoma is $74.1 \%$ and $92.9 \%$ for blacks and whites, respectively; notably accurate staging was not well established in this study [3]. However, the five-year survival varies from $>90 \%$ in stage la, to $<30 \%$ in stage IV [4]. Distribution in acral areas is a common presentation in darkly-pigmented patients, and is often detected late, which may explain the worse prognosis for black patients [5].

The clinical presentation of melanoma varies largely on its type, of which there are four broad classifications: (1) superficial spreading melanoma; (2) nodular melanoma; (3) lentigo maligna; and (4) acral lentiginous. In general, a dermatology referral is indicated if a post-pubertal or longstanding nevus changes in shape, colour, or size; any nevi with three or more colours; a nevus that itches or bleeds; a new persistent skin lesion emerges, especially if pigmented or vascular in appearance; or a pigmented line in a nail or a lesion under a nail develops, especially if there is no history of trauma [6]. The ABCDEs of melanoma (asymmetry, border irregularities, multiple or dark colours, diameter $\geq 6 \mathrm{~mm}$, and evolution over time) are classically used to diagnose suspicious lesions, but the "ugly duckling sign" has recently become a surrogate for this method. The "ugly ducking sign" establishes suspicion by evaluating a patient's personal nevi pattern and identifying outliers [7].

We present the case report of a 63-year-old white man who presented to his family doctor with a history of axillary lymphadenopathy and a past medical history of melanoma. Lymph node biopsies demonstrated malignant melanoma.

\section{CASE PRESENTATION}

A 35-year-old white man was referred to a dermatologist with a 6-week history of a quickly evolving, bleeding nevus on the dorsal aspect of his right forearm. The nevus was asymptomatic, and he was otherwise healthy. Chronic exposure to sun with an-

Keywords: Melanoma; Chemotherapy; Immunotherapy; Management; Multidisciplinary 


\section{Case Report}

nual "burning then tanning" as a child was elicited on history. In fact, one of the patient's sunburns was "so bad he sat in chills for the entire night." Although he had blond hair, he noted a strong family history of lentigines and red hair. The nevus was excised with wide margins. Dermatopathology revealed a stage 2a superficial spreading melanoma with clear margins. The area was re-excised, as is the standard of care. At this time, the patient was considered cured and was educated regarding sun exposure and the prevention of melanoma.

Twenty-eight years later, the patient noticed a lump in his right axilla when applying deodorant and proceeded to visit his family doctor who ordered an ultrasound, followed by an ultrasoundguided biopsy. The patient was diagnosed with metastatic melanoma two weeks later. After being consulted about his treatment options, he decided to undergo a right axillary lymph node dissection. The lymph node dissection showed 7 nodes with cancerous cells, and a PET scan elucidated two previously unknown metastatic nodules in his spleen. A splenectomy was performed within two months, and he subsequently underwent adjuvant radiation followed by chemotherapy (dacarbazine). The radiation treatment was directed at the dissected axillary and contiguous supraclavicular nodal regions. $6 \mathrm{MV}$ photons 54Gy in 27 fractions / 5 fractions per week over 5.5 weeks were delivered. Bolus was used to ensure full dose to the axillary scar. Chemotherapy was discontinued shortly thereafter, as it was deemed ineffective, and ipilimumab was started. Ipilimumab is a first-inclass T-cell potentiator, which has shown significant benefit in progression-free survival and durable remission when compared to conventional treatment strategies for stage IV melanoma [8]. Our patient received four courses of ipilimumab; this treatment regime was completed within three months. He then underwent three PET scans, all of which showed no evidence of metastatic disease. Under the advice of his oncologist, he began serial chest CT scans after three months reprieve, the first of which showed evidence of metastatic disease.

Currently, other treatment options have been discussed, but not yet initiated. The options are: mono-immunotherapy anti-PD-1 treatment with nivolumab (Opdivo) or pembrolizumab (Keyturda); combination therapy with either nivolumab or pembrolizumab and ipilumumab; or, BRAF v600e mutation targeted treatment with vemurafenib or dabrafenib, given the BRAF v600e mutation present in his melanoma. An application to fund combination therapy for his unique circumstance is currently pending. Public funding for his proposed treatment exists only as a first line compassionate measure in patients who have not previously received ipilimumab; his previous immunotherapy disqualifies him for coverage under this provision.

Lifestyle modifications must also be mentioned. Given his previous illness, our patient increased his sun protection by using SPF
60 (UVA/UVB) sunscreen, sporting long sleeve t-shirts and pants, wearing a large hat, and avoiding peak ultraviolet radiation hours; it was life-changing in this case for the patient to know that sun exposure is cumulative and does not reset to zero. Prior to his chemotherapy, the patient began exercising (running, walking), eating healthily, adopting a positive outlook and a healthy sleep schedule. Alternative medicine providers (Naturopaths, Osteopath, Physiotherapist) have helped facilitate his lifestyle changes and psychological stress management, creating in him a sense of emotional well-being, which he hopes will increase the efficacy of his medical management.

\section{DISCUSSION}

Melanoma is a common and potentially lethal skin cancer; although it is most commonly found in sun-exposed regions, it may appear on any skin surface, especially in those with darker pigmentation, as well as in other places such as the eye and central nervous system. Over $90 \%$ of melanomas present in those 10 years of age or older [9] and rarely present in childhood or adolescence, with the incidence only increasing in those 20 years of age or older [10]. Morphologically superficial spreading melanoma, nodular melanoma, lentigo maligna, and acral melanoma are the most common; however, amelonotic melanoma and other sub categories do exist. As mentioned, melanomas typically present as quickly evolving, polychromatic, atypical nevi, which may ulcer or bleed when more advanced [7].

Our patient's case is a classic presentation with expected risk factors. A young, fair skinned male with a history of blistering sunburns as a child presented with a rapidly evolving atypical mole, which proved to be melanoma. On average, $16 \%$ of lymph node negative melanomas recur within 61 months, after which a patient is considered cured [11]. It is extremely uncommon for melanoma to relapse later than 5 years after the primary lesion, but it has been shown that men are at an increased risk for recurrence with long-term follow-up ( $\geq 15$ years later) [12]. Our case demonstrates this with a 28 -year delay in recurrence.

Primary and secondary prevention involves taking personal accountability for cumulative sun exposure, especially in demographically high-risk patients. Increasing safe sun practices (SPF 60 UVB/UVA, long sleeves/large hats, avoiding peak sun hours from 10:00 a.m. to 2:00 p.m.) is pivotal in the prophylactic management of melanoma. Any sun exposure or exposure to ultraviolet light may increase the possibility of primary melanoma in at-risk patients [13].

If prevention is not successful, a multidisciplinary approach must be employed in the management of melanoma, as well as an assessment of how each variable increases survivorship and/ or quality of life. In our patient's case, the use of naturopathic 
medicine, physiotherapy, exercise, optimal nutrition, and positive stress management have all played at least a subjective role in the quality of his life; it was important for him to establish a personalized treatment regime to alleviate stressors and improve his well-being. As anecdotal as one account remains, the psychological consequences of cancer treatment are well recognized, as is the utility of alternative medicines in alleviating this burden [14]. Synergistically, we recognize the efficacy of westernized, evidence-based medicine and alternative medicine in the holistic management of melanoma.

Surgical management used in our patient included excision of the primary melanoma with wide margins (and dermatopathology), lymph node dissection of axillary metastasis, and a splenectomy for splenic metastasis. Furthermore, local adjuvant radiation therapy, general chemotherapy, and targeted immunologics were implemented for curative measures. Adjuvant radiation therapy has been demonstrated in a Phase III trial to reduce regional nodal recurrence and improve disease-free survival when administered to patients judged to be at high risk for regional failure on the basis of resected nodal size, number of involved nodes, and presence of extracapsular spread. The clinical tradeoff is the acute radiation dermatitis that is generally shortlived but the more significant $15-25 \%$ risk of extremity lymphedema that results from the radiation fibrosis superimposed on surgical lymphatic extirpation [15]. Ipilimumab is a recombinant human IgG1 monoclonal antibody, which binds to cytotoxic T-lymphocyte associated antigen 4 (CTLA-1) blocking its pathway; this results in T-cell potentiation, which may augment the body's immunological attack against melanoma [16]. The eventual use of nivolumumab (or its equivalent pembrolizumab) will occur in our case. Nivolumumab is an IgG4 subclass program cell death 1 (PD1) inhibiting antibody, which prevents the breakdown of PD-1 expressing T cells, B cells, and NK cells; thus potentiating the body's immune response to the melanoma. This therapy has prolonged survival in those with metastatic melanoma when used as a mono-therapy or in combination with ipilimumab $[17,18]$. In fact, beneficial outcomes were shown in melanomas irrespective of their PD-1 status (positive or negative) [18], but these treatments are not without risks. While the progression-free survival was greater in patients who received combination therapy, so were the risks of serious complications ( $54 \%$ for combination, and $24 \%$ for mono-therapy) [19]. Serious complications are classified as grade 3 or grade 4 (severe or life-threatening) and range in frequency with colitis, elevated alanine aminotransferase, and diarrhea being the most common [19]; it is noteworthy that 25-30\% of the patients included in studies continued treatment, despite grade 3-4 complications. Lastly, genomic PCR for BRAF v600e, a common mutation in melanoma, and present in our case, makes accessible future avenues for treatment with targeted therapies. Vemurafenib, a BRAF kinase inhibitor and inhibitor of v600e, or Dabrafenib, a more selective inhibitor of mutated protein ki- nase BRAF (including v600e), have shown promise and remain possible recourses should our patient's current management be unsuccessful $[20,21]$. Patients treated with vemurafenib, despite the short duration of responses, have a high overall response rate and frequently experience dramatic tumor regression $(>80 \%$ targeted inhibition is required for a clinical response) (22). Further work in the field to establish the utility of targeted therapy, in combination with other chemotherapies or immune modulators, has also shown promising results $[23,24]$ and furthers our hope for an eventual cure.

\section{CONCLUSIONS}

Patients with metastatic melanoma may present in numerous morphologically distinct manners, which reduce in incidence when preventative measures are taken. Risk stratification is required to increase awareness of patients and their relatives. The management of melanoma, although surgical for early-stage cases, is multidisciplinary, patient-dependent, and potentially targeted depending on genetic factors. It is crucial to evaluate the impact of a patient's diagnosis on medical, physical, psychological, and spiritual well-being to increase survival rates and improve quality of life.

\section{CONSENT}

Written informed consent was obtained from the patient for the publication of this case report. The care and reporting conform fully to the Helsinki Declaration.

\section{REFERENCES}

1. Kohler BA, Sherman RL, Howlader N, et al. Annual report to the nation on the status of cancer, 1975-2011, featuring incidence of breast cancer subtypes by race/ethnicity, poverty, and state. J Natl Cancer Inst. 2015;107(6):djv048.

2. Melanoma Statistics [Internet]. Canadian Cancer Statistics 2015. 2015 [cited 2016 Mar 13]. Available from: http://www.cancer.ca/en/cancer-information/cancer-type/skin-melanoma/statistics/?region=on.

3. Balch CM, Soong SJ, Gershenwald JE, et al. Prognostic factors analysis of 17,600 melanoma patients: validation of the American Joint Committee on Cancer melanoma staging system. J Clin Oncol. 2001;19(16):3622-3634.

4. Balch C, Gershenwald J, Soong S, et al. Final Version of 2009 AJCC Melanoma Staging and Classification [Internet]. American Society of Clinical Oncology. 2009. Available from: http://jco.ascopubs.org/content/27/36/6199.full.

5. Shoo BA, Kashani-Sabet M. Melanoma arising in African-, Asian-, Latino- and Native-American populations. Semin Cutan Med Surg. 2009;28(2):96-102.

6. Marsden JR, Newton-Bishop JA, Burrows L, et al. Revised U.K. guidelines for the management of cutaneous melanoma 2010. Br J Dermatol. 2010;163(2):238-256.

7. Gachon J, Beaulieu P, Sei JF, et al. First prospective study of the recognition process of melanoma in dermatological practice. Arch Dermatol. 2005;141(4):434-438.

8. Tarhini A, Lo E, Minor DR. Releasing the brake on the immune system: ipilimumab in melanoma and other tumors. Cancer Biother Radiopharm. 2010;25(6):601-613.

9. Lange JR, Palis BE, Chang DC, Soong S-J, Balch CM. Melanoma in children and teenagers: an analysis of patients from the National Cancer Data Base. J Clin Oncol. 2007;25(11):1363-1368.

10. Strouse JJ, Fears TR, Tucker MA, Wayne AS. Pediatric melanoma: risk factor and survival analysis of the surveillance, epidemiology and end results database. J Clin Oncol. 2005;23(21):4735-4741. 


\section{Case Report}

11. Jones EL, Jones TS, Pearlman NW, et al. Long-term follow-up and survival of patients following a recurrence of melanoma after a negative sentinel lymph node biopsy result. JAMA Surg. 2013;148(5):456-461.

12. Tsao $H$, Cosimi AB, Sober AJ. Ultra-late recurrence (15 years or longer) of cutaneous melanoma. Cancer. 1997;79(12):2361-2370.

13. Newton-Bishop JA, Chang Y-M, Elliott F, et al. Relationship between sun exposure and melanoma risk for tumours in different body sites in a large case-control study in a temperate climate. Eur J Cancer. 2011;47(5):732741.

14. Paul A, Cramer H, Lauche R, Altner N, Langhorst J, Dobos GJ. An oncology mind-body medicine day care clinic: concept and case presentation. Integr Cancer Ther. 2013;12(6):503-507.

15. Henderson MA, Burmeister B, Thompson JF, et al. Adjuvant radiotherapy and regional lymph node field control in melanoma patients after lymphadenectomy: Results of an intergroup randomized trial. J Clin Oncol. 2009;27(18s).

16. Wolchok JD, Neyns B, Linette G, et al. Ipilimumab monotherapy in patients with pretreated advanced melanoma: a randomised, double-blind, multicentre, phase 2, dose-ranging study. Lancet Oncol. 2010;11(2):155-164.

17. Topalian SL, Sznol M, McDermott DF, et al. Survival, durable tumor remission, and long-term safety in patients with advanced melanoma receiving nivolumab. J Clin Oncol. 2014;32(10):1020-1030.

18. Larkin J, Chiarion-Sileni V, Gonzalez R, et al. Combined nivolumab and ipilimumab or monotherapy in untreated melanoma. N Engl J Med. 2015;373(1):23-34.

19. Postow MA, Chesney J, Pavlick AC, et al. Nivolumab and ipilimumab versus ipilimumab in untreated melanoma. N Engl J Med. 2015;372(21):20062017.

20. Chapman PB, Hauschild A, Robert C, et al. Improved survival with vemurafenib in melanoma with BRAF V600E mutation. $N$ Engl J Med. 2011;364(26):2507-2516.

21. Flaherty $\mathrm{KT}$, Robert $\mathrm{C}$, Hersey $\mathrm{P}$, et al. Improved survival with MEK inhibition in BRAF-mutated melanoma. N Engl J Med. 2012;367(2):107-114.

22. Holderfield M, Deuker MM, McCormick F, McMahon M. Targeting RAF kinases for cancer therapy: BRAF-mutated melanoma and beyond. Nat Rev Cancer. 2014;14(7):455-467.

23. Robert C, Karaszewska B, Schachter J, et al. Improved overall survival in melanoma with combined dabrafenib and trametinib. N Engl J Med. 2015;372(1):30-39.

24. Vennepureddy A, Thumallapally N, Motilal Nehru V, Atallah J-P, Terjanian T. Novel drugs and combination therapies for the treatment of metastatic melanoma. J Clin Med Res. 2016;8(2):63-75. 